\title{
SOME RESULTS USING GENERAL MOMENT FUNCTIONS
}

\author{
WALTER L. SMITH ${ }^{1}$
}

(Received 5 December 1967; revised 27 January 1969)

\begin{abstract}
Summary: Let $\left\{X_{n}\right\}$ be a sequence fo independent and identically distributed random variables such that $0<\mu=\mathscr{E} X_{n} \leqq+\infty$ and write $S_{n}=X_{1}+X_{2}+\ldots+X_{n}$. Let $v \geqq 0$ be an integer and let $M(x)$ be a non-decreasing function of $x \geqq 0$ such that $M(x) / x$ is non-increasing and $M(0)>0$. Then if $\mathscr{E}\left|X_{\mathbf{1}}\right| \boldsymbol{v}_{M}\left(\left|X_{1}\right|\right)<\infty$ and $\mu<\infty$ it follows that $\mathscr{E}\left|S_{n}\right|^{\nu} M\left(\left|S_{n}\right|\right) \sim(n \mu)^{\nu} M(n \mu)$ as $n \rightarrow \infty$. If $\mu=\infty(\nu=0)$ then $\mathscr{E} M\left(\left|S_{n}\right|\right)=0(n)$. A variety of results stem from this main theorem (Theorem 2), concerning a closure property of probability generating functions and a random walk result (Theorem 1) connected with queues.
\end{abstract}

\section{Introduction}

The work of the present note originated in a desire to prove Theorem 1 below, which, in a somewhat more restricted version, was announced by the author during the Chapel Hill Symposium on Congestion Theory (Smith and Wilkinson (1965), pp. 132-133). The proof then available was needlessly complicated; it will now follow trom a useful general theorem on generating functions (Corollary 3.1, below).

In Smith (1967) a class $\mathscr{M}$ of 'moment functions' $M(x)$ is introduced as a means of dealing with problems involving a random variable $X$, say, when it is given that the highest absolute moment of $X$ known to be finite is not necessarily an integral power of $X$. For example, one might be given that $\mathscr{E}|X|^{\frac{3}{2}} \log ^{+}|X|<\infty$. In Smith (1967) the use of such information in the expansion of characteristic functions and in certain renewal-theoretic problems is discussed. A rather more fundamental property of a large class of these functions $M$ is as follows. If $X_{1}, X_{2}, \ldots$, are independent and identically distributed random variables such that $0<\mu=\mathscr{E} X_{n}<\infty$ and if $S_{n}=X_{1}+\ldots+X_{n}$, then provided $\mathscr{E} M(|X|)<\infty$ it follows that $\mathscr{E} M\left(\left|S_{n}\right|\right) \sim M(n \mu)$ as $n \rightarrow \infty$. This result is described in detail in Theorem 2 .

Theorem 3, below, is then an easy consequence of Theorem 2 . It concerns the finiteness of $\mathscr{E} M\left(\left|S_{N}\right|\right)$ when $N$ is a non-negative integer-valued random variable independent of the $\left\{X_{n}\right\}$. When the $\left\{X_{n}\right\}$ are themselves

1 This research was supported by the Department of the Navy, Office of Naval Research. Grant NONR-855(09). 
also non-negative integer-valued random variables we are led at once to Corollary 3.1, below, conceining a useful closure property of probability generating functions. As explained, it is from this Corollary that we deduce Theorem 1, the original object of the present work. ${ }^{2}$

We now state:

TheOREM 1. (j) Let $\left\{X_{n}\right\}$ be an infinite sequence of independent and identically distributed random variables, with common distribution function $F(x)=P\left\{X_{n} \leqq x\right\}$, such that $0<\mathscr{E} X_{n} \leqq+\infty$.

(ii) Write $S_{n}=X_{l}+\ldots+X_{n}$, as usual, and $T_{n}=\max \left\{S_{1}, S_{2}, \ldots, S_{n}\right\}$.

(iij) Let $v \geqq 0$ be un integer and $M(x)$ be a non-decreasing function of $x \geqq 0$ such that $M(x) / x$ is non-increasing for all large $x$. Suppose $M(0)>0$ (and note that $M(x)$ may be a constant identically). Then any one of the following three statements implies the other two:

$$
\begin{aligned}
& \int_{-\infty}^{0}|x|^{(\nu+1)} M(|x|) d F(x)<\infty ; \\
& \sum_{n=1}^{\infty} n^{(\nu-1)} M(n) P\left\{S_{n} \leqq 0\right\}<\infty ; \\
& \sum_{u=1}^{\infty} n^{\nu} M(n) P\left\{T_{n} \leqq 0\right\}<\infty .
\end{aligned}
$$

In order to prove this result on random walks, which incidentally is of some relevance to the theory of queues, we prove the following Theorem 2. This theorem would appear to be of general interest.

Theorem 2. Suppose the conditions and notations (i), (ii) and (iii) of Theorem 1 hold, with the additional proviso that $\mu=\mathscr{E} X_{n}<\infty$.

Then, if $\mathscr{E}\left|X_{1}\right|^{\nu} M\left(\left|X_{1}\right|\right)<\infty$, it followes that, as $n \rightarrow \infty$,

$$
\mathscr{E}\left|S_{n}\right|^{\nu} M\left(\left|S_{n}\right|\right) \sim(n \mu)^{\nu} M(n \mu) .
$$

If, however, $\mu=\mathscr{E} X_{n}=\infty$ (which can be the case only when $\nu=0$ ) one can infer from $\mathscr{E} M\left(\left|X_{1}\right|\right)<\infty$ only the weaker conclusion. $\mathscr{E} M\left(\left|S_{n}\right|\right)=0(n)$.

From Theorem 2, as already explained, we can easily deduce the following theorem concerning moments of sums of random numbers of random variables.

THEOREM 3. (i) Let $\left\{X_{n}\right\}$ be an infinite sequence of independent and identically distributed random variables such that $0<\mathscr{E} X_{n}<\infty$ and rerite $B_{n}(x)=P\left\{S_{n} \leqq x\right\}$ for $n=1,2, \ldots ; B_{0}(x)=P\{0 \leqq x\}$.

2 The special case of this theorem when $M$ is a constant was obtained by Heyde (1964). 
(ii) Let $\left\{c_{n}\right\}$ be $x$ discrete probability distribution (i.e. $c_{n} \geqq 0$, all $n$, and $\left.\sum_{0}^{\infty} c_{n}=1\right)$ such that $c_{0}<1$.

(iii) Let the d.f. $A(x)$ be thus defined:

$$
A(x)=\sum_{n=0}^{\infty} c_{n} B_{n}(x)
$$

(iv) Let $\nu$ and $M(x)$ be as in Theorem 1 (iii).

Then a necessary and sufficient condition to ensure that

$$
\int_{-\infty}^{+\infty}|x|^{v} M(|x|) d A(x)<\infty
$$

is that both the following statements be true:

$$
\begin{aligned}
& \int_{-\infty}^{+\infty}|x|^{\nu} M(|x|) d B_{1}(x)<\infty ; \\
& \sum_{n=1}^{\infty} n^{\nu} M(n) c_{n}<\infty .
\end{aligned}
$$

$I f$, for the case $v=0$, it should happen that (i), ..., (iv) hold except for the fact that $\mathscr{E} X_{n}=+\infty$ then one can still infer that (1.4) is true if (1.5) is given, together with the condition $\sum_{1}^{\infty} n c_{n}<\infty$.

If $v$ and $M(x)$ are as in Theorem 1 and if $\Pi(s)=\sum \pi_{n} s^{n}, 0 \leqq s \leqq 1$, is a probability generating function, then we shall say that $\Pi$ is in the class $\mathscr{G}(v, M)$ if

$$
\sum_{n=1}^{\infty} n^{\nu} M(n) \pi_{n}<\infty
$$

An immediate corollary to Theorem 3 is the following:

Corollary 3.1. Let $v$ and $M(x)$ be as in Theorem 1 (iii) and let $\alpha(s)$, $\beta(s), \gamma(s)$ be probability generating functions, $0 \leqq s \leqq 1$. Suppose that $\beta(s)=\sum_{0}^{\infty} b_{n} s^{n}$, that $\sum_{0}^{\infty} n b_{n}<\infty$, and that $\alpha(s)=\gamma(\beta(s))$. Then $\alpha \in \mathscr{G}(v, M)$ if and only if both $\beta$ and $\gamma$ belong to $\mathscr{G}(\nu, M)$.

If, for the case $v=0$, it should happen that $\sum_{1}^{\infty} n b_{n}=\infty$ one can still infer that $\alpha \in \mathscr{G}(0, M)$ if it is given that $\beta \in \mathscr{G}(0, M)$ and $\sum_{1}^{\infty} n c_{n}<\infty$.

We close this introduction by mentioning that in the course of proving Theorem 2 we establish the following:

COROLLARY 2.1. Under the conditions of Theorem 1, for any $\varepsilon>0$,

$$
\int_{\left|\frac{S_{n}}{n}-\mu\right|>\varepsilon}\left|S_{n}\right|^{\nu} M\left(\left|S_{n}\right|\right) d P=0\left(n^{\nu} M(n)\right),
$$

as $n \rightarrow \infty$.

Needless to say, every result in this paper which depends on the 
condition $0<\mathscr{E} X_{n} \leqq \infty$ has a counterpart when $-\infty \leqq \mathscr{E} X_{n}<0$; the essential requirement being $\mathscr{E} X_{n} \neq 0$, of course.

\section{On moment functions and some preliminary lemmas}

In Smith (1967) we have introduced a class $\mathscr{M}$ of moment-functions. A function $M(x)$ belongs to $\mathscr{M}$ if: (i) it is non-decreasing in $[0, \infty)$; (ii) $M(x) \geqq 1$, all $x \geqq 0$; (iii) $M(x+y) \leqq M(x) M(y)$ for all $x, y \geqq 0$. If $M \in \mathscr{M}$ and, additionally, (iv) $M(2 x)=0(M(x))$ for all $x \geqq 0$ then we say $M \in^{\prime} \mathscr{M}^{*}$. We say a d.f. $F(x)$ is in the class $\mathscr{D}(\nu, M)$, for any real $\nu \geqq 0$, if $\int_{-\infty}^{+\infty}|x|^{v} M(|x|) d F(x)<\infty$. From the point of view of defining a class $\mathscr{D}(v, M)$, the moment function $M(x)$ can be replaced by any non-decreasing

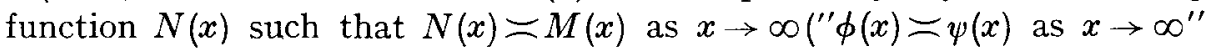
means that there exist positive constants $\delta$ and $\Delta$ such that $\delta \phi(x) \leqq$ $\psi(x) \leqq \Delta \phi(x)$ for all large $x)$. Thus we are led to introduce an extended class $E \mathscr{M}^{*}$ in such a way that a function $N(x) \in E \mathscr{M}^{*}$ if we can find a function $M(x) \in \mathscr{M}^{*}$ such that $N(x)=M(x)$ as $x \rightarrow \mid \infty$. We can then say $M(x)$ represents $N(x)$ in $\mathscr{M}^{*}$.

Suppose that for some $\Delta \geqq 0$ and some $A>0$ the function $N(x)$ satisfies the following:

(i) $N(x)$ is non-decreasing for $x \geqq \Delta$;

(ii) $N(\Delta)>0$;

(iii)' $N(x+y) \leqq A N(x) N(y)$ for all $x \geqq \Delta, y \geqq \Delta$;

(iv)' $N(2 x)=0(N(x))$ for all $x \geqq \Delta$.

Then we may suppose, with no less of generality, that $A N(A) \geqq 1$ and define

$$
\begin{aligned}
M(x) & =A N(x), \quad x \geqq \Delta, \\
& =M(\Delta), \quad x \leqq \Delta ;
\end{aligned}
$$

the function $M(x)$ so defined belongs to $\mathscr{M}^{*}$. Thus a function $N(x)$ satisfying (i)', (ii)', (iii)' and (iv)' above belongs to $E \mathscr{M}^{*}$.

Now suppose that, for some $\Delta \geqq 2, M(x)$ is non-decreasing and $M(x) / x$ is non-increasing for all $x \geqq A$, and $M(\Delta)>0$. Let $v \geqq 0$ be a constant, and define $N_{\nu}(x)=x^{\nu} M(x)$ for all $x \geqq \Delta$. It is clear that (i)' and (ii)' above are satisfied by $N_{\nu}(x)$. Further, if $x \geqq \Delta$ and $y \geqq \Delta$,

$$
\frac{N_{\nu}(x+y)}{N_{\nu}(x) N_{\nu}(y)}=\frac{(x+y)^{\nu}}{x^{\nu} y^{\nu}} \cdot \frac{(x+y)}{M(x) M(y)}\left\{\frac{M(x+y)}{x+y}\right\} .
$$

But, since $M(x) / x$ is non-increasing,

so that

$$
(x+y)\left\{\frac{M(x+y)}{(x+y)}\right\} \leqq x\left\{\frac{M(x)}{x}\right\}+y\left\{\frac{M(y)}{y}\right\}
$$




$$
\begin{aligned}
\frac{N_{\nu}(x+y)}{N_{\nu}(x) N_{\nu}(y)} & \leqq\left(\frac{1}{x}+\frac{1}{y}\right)^{\nu}\left\{\frac{1}{M(x)}+\frac{1}{M(y)}\right\} \\
& \leqq \frac{2}{M(\Delta)},
\end{aligned}
$$

since $x \geqq \Delta, y \geqq \Delta$. Thus $N(x)$ satisfies (iii)' above. Finally, for $x \geqq \Delta$,

$$
\begin{aligned}
\frac{N_{\nu}(2 x)}{N_{\nu}(x)} & \leqq 2^{v} \frac{M(2 x)}{M(x)} \\
& =2^{(\nu+1)} \frac{x}{M(x)}\left\{\frac{M(2 x)}{2 x}\right\} \\
& \leqq 2^{(\nu+1)} \frac{x}{M(x)}\left\{\frac{M(x)}{x}\right\}
\end{aligned}
$$

Thus $N_{\nu}(x)$ also satisties (iv)' and so belongs to $E \mathscr{M}^{*}$ and there will be a function $M_{\nu}(x) \in \mathscr{M}^{*}$ which represents $N_{\nu}(x)$. We shall make frequent use of these functions $M_{\nu}(x)$.

With these preliminaries set aside we can now prove the following.

Lемма 2.1. Let $k \geqq 1$ be an integer and let $M(x) \in \mathscr{M}$. Suppose $X_{1}$, $X_{2}, \ldots, X_{k}$ are independent and identically distributed random variables and write $S_{j}=X_{1}+\ldots+X_{j}$ for $j=1,2, \ldots, k$. Then $\mathscr{E} M\left(\left|S_{k}\right|\right)<\infty$ if and only if $\mathscr{E} M\left(\left|X_{1}\right|\right)<\infty$.

Proof. Clearly $M\left(\left|S_{k}\right|\right) \leqq M\left(\sum_{1}^{k}\left|X_{j}\right|\right)$, and so, by a property of functions in $\mathscr{M}$,

Therefore

$$
M\left(\left|S_{k}\right|\right) \leqq \prod_{j=1}^{k} M\left(\left|X_{j}\right|\right)
$$

$$
\mathscr{E} M\left(\left|S_{k}\right|\right) \leqq\left[\mathscr{E} M\left(\left|X_{1}\right|\right)\right]^{k},
$$

and part of the lemma is proved.

If $P\left\{X_{1} \geqq 0\right\}=1$, or if $P\left\{X_{1} \leqq 0\right\}=1$ the rest of the proof is trivial. Suppose therefore that both $P\left\{X_{1} \geqq 0\right\}>0$ and $P\left\{X_{1}<0\right\}>0$. Then both $P\left\{S_{k-1} \geqq 0\right\}>0$ and $P\left\{S_{k-1}<0\right\}>0$. The finiteness of $\mathscr{E} M\left(\left|S_{k}\right|\right)$ then implies that, for example,

$$
\mathscr{E}\left\{M\left(\left|S_{k}\right|\right) \mid S_{k-1}<0 ; X_{k}<0\right\}
$$

is also finite. However, if both $S_{k-1}$ and $X_{k}$ are negative then $\left|X_{k}\right|<\left|S_{k}\right|$ and so

$$
\mathscr{E}\left\{M\left(\left|X_{k}\right|\right) \mid S_{k-1}<0 ; X_{k}<0\right\}
$$

must be finite. But $X_{k}$ and $S_{k-1}$ are independent. Thus $\mathscr{E}\left\{M\left(\left|X_{k}\right|\right) \mid X_{k}<0\right\}$ 
is finite. Similarly one can deal with $\mathscr{E}\left\{M\left(\left|X_{k}\right|\right) \mid X_{k} \geqq 0\right\}$, and the lemma follows.

LEMmA 2.2. If, for large $x, L(x)$ is non-increasing and $x L(x)$ is nondecreasing, then

and

$$
\lim _{\varepsilon \downarrow 0+} \limsup _{n \rightarrow \infty} \frac{L(\overline{1+\varepsilon} n)}{L(n)}=1
$$

$$
\lim _{\varepsilon \downarrow 0+} \liminf _{n \rightarrow \infty} \frac{L(\overline{1+\varepsilon n})}{L(n)}=1 .
$$

Proof. If we set $M(x)=x L(x)$, then

which implies that for all $n$

$$
\frac{M(\overline{1+\varepsilon} n)}{M(n)} \geqq 1
$$

$$
1 \geqq \frac{L(\overline{1+\varepsilon} n)}{L(n)} \geqq \frac{1}{1+\varepsilon} .
$$

The lemma follows at once from these inequalities.

\section{Proof of Theorem 2}

Let us first assume that $0<\mu<\infty$, and postpone discussion of the case $\mu=\infty$.

Let us write $M_{v}(x), v>0$, for any member of $\mathscr{M}^{*}$ such that $M_{\nu}(x)=x^{\nu} M(x)$ as $x \rightarrow \infty$. Suppose that the integer $v \geqq 1$. Then we can find a $K_{1}>0, K_{2}>0$, such that, for all large $x$,

$$
\begin{aligned}
M_{\nu}(x) & \leqq K_{1} x M_{\nu-1}(x) \\
& \geqq K_{2} x M_{\nu-1}(x) .
\end{aligned}
$$

For large $R>0$ and small $\varepsilon>0$, define events

$$
\begin{gathered}
A_{n}(\varepsilon) \equiv\left\{\left|S_{n}\right| n-\mu \mid \leqq \varepsilon\right\} \\
B_{n}(R) \equiv\left\{\left|S_{n}\right| n-\mu \mid>R\right\} .
\end{gathered}
$$

Further, for $n=1,2, \cdots$, let

Then

$$
\Delta_{\nu}(R, n)=\int_{B_{n}(R)} \frac{M_{\nu}\left(\left|S_{n}\right|\right)}{M_{\nu}(n \mu)} d P .
$$

$$
\begin{aligned}
\Delta_{\nu}(R, n) & \leqq \frac{K_{1}}{K_{2} n \mu} \int_{B_{n}(R)}\left|S_{n}\right| \frac{M_{\nu-1}\left(\left|S_{n}\right|\right)}{M_{\nu-1}(n \mu)} d P \\
& \leqq \frac{K_{1}}{K_{2} \mu} \int_{B_{n}(R)}\left|X_{n}\right| \frac{M_{\nu-1}\left(\left|S_{n}\right|\right)}{M_{\nu-1}(n \mu)} d P,
\end{aligned}
$$


by symmetry and the inequality $\left|S_{n}\right| \leqq\left|X_{1}\right|+\cdots+\left|X_{n}\right|$. But

$$
\begin{aligned}
M_{v-1}\left(\left|S_{n}\right|\right) & \leqq M_{\nu-1}\left(\left|S_{n-1}\right|+\left|X_{n}\right|\right) \\
& \leqq M_{\nu-1}\left(\left|X_{n}\right|\right) M_{\nu-1}\left(\left|S_{n-1}\right|\right),
\end{aligned}
$$

by a defining property of $\mathscr{M}^{*}$. Hence we have

$$
\begin{aligned}
\Delta_{\nu}(R, n) \leqq & \frac{K_{\mathrm{J}}}{K_{2}^{2} \mu} \int_{B_{n}(R)} M_{\nu}\left(\left|X_{n}\right|\right) \frac{M_{\nu-1}\left(\left|S_{n-1}\right|\right)}{M_{\nu-1}(n \mu)} d P \\
\leqq & \frac{K_{1}}{K_{2}^{2} \mu} \int_{\left|S_{n-1}-(n-1) \mu\right|>\frac{1}{2} n R} \\
& +\frac{K_{1}}{K_{2}^{2} \mu} \int_{\left|X_{n}-\mu\right|>\frac{1}{2} n R} \\
= & I_{1}(n)+I_{2}(n), \text { say. }
\end{aligned}
$$

Because $X_{n}$ and $S_{n-1}$ are independent we have

$$
I_{2}(n) \leqq \frac{K_{1} \mathscr{E} M_{v-1}\left(\left|S_{n-1}\right|\right)}{K_{2}^{2} \mu M_{\nu-1}(n \mu)} \int_{\left|X_{1}-\mu\right|>\frac{1}{2} n R} M_{v}\left(\left|X_{1}\right|\right) d P,
$$

using the fact that $X_{1}$ and $X_{n}$ are identically distributed. In a similar way we find

$$
I_{1}(n) \leqq \frac{K_{1} \mathscr{E} M_{\nu}\left(\left|X_{1}\right|\right)}{K_{2}^{2} \mu} \Delta_{\nu-1}\left(\frac{1}{2} R, n-1\right) .
$$

For integer $\lambda \geqq 0$, let $\mathscr{H}_{\lambda}$ be the hypothesis that the following two statements are true:

(i) $\mathscr{E} M_{\lambda}\left(\left|S_{n}\right|\right)=0\left(M_{\lambda}(n \mu)\right)$, as $n \rightarrow \infty$;

(ii) For all sufficiently large $R, \Delta_{\lambda}(R, n) \rightarrow 0$, as $n \rightarrow \infty$.

The inequalities (3.2), (3.3) and (3.4) show that if $\mathscr{E} M_{\nu}\left(\left|X_{1}\right|\right)$ is finite and if $\mathscr{H}_{(\nu-1)}$ is true, then

$$
\Delta_{v}(R, n) \rightarrow 0 \text {, as } n \rightarrow \infty,
$$

for all large $R$. Furthermore,

$$
\frac{\mathscr{E} M_{\nu}\left(\left|S_{n}\right|\right)}{M_{\nu}(n \mu)} \leqq \frac{M_{\nu}(\overline{R+\mu} n)}{M_{\nu}(\mu n)}+\Delta_{\nu}(R, n) .
$$

But, for some $K_{4}>0$,

$$
\begin{aligned}
\frac{M_{\nu}(\overline{R+\mu} n)}{M_{\nu}(\mu n)} & \leqq \frac{K_{4}(R+\mu)^{\nu} M(\overline{R+\mu} n)}{\mu^{\nu} M(\mu n)} \\
& \leqq \frac{K_{4}(R+\mu)^{\nu+1}}{\mu^{\nu+1}}
\end{aligned}
$$


since $M(x) / x$ is non-increasing

From (3.5), (3.6) and (3.7) we see that

$$
\mathscr{E} M_{\nu}\left(\left|S_{n}\right|\right)=0\left(M_{\nu}(n \mu)\right) \text {, as } n \rightarrow \infty \text {. }
$$

Thus $\mathscr{H}_{\nu}$ is true if $\mathscr{H}_{(\nu-1)}$ is true and $\mathscr{E} M_{\nu}\left(\left|X_{1}\right|\right)<\infty$. Plainly we desire to show that $\mathscr{H}_{0}$ is true, from which it would follow (if $\mathscr{E} M_{v}\left(\left|X_{1}\right|\right)<\infty$ ) that $\mathscr{H}_{\nu}$ is true.

Let us write $M(x)=x L(x)$, where $L(x)$ is a non-increasing function of $x$. Then

$$
\begin{aligned}
\Delta_{0}(R, n) & =\int_{B_{n}(R)} \frac{\left|S_{n}\right| L\left(\left|S_{n}\right|\right)}{n \mu L(n \mu)} d P \\
& \leqq \frac{L(\overline{R-\mu n})}{\mu L(n \mu)} \int_{B_{n}(R)}\left|\frac{S_{n}}{n}\right| d P .
\end{aligned}
$$

Let us define, in a familiar way, for $n=1,2, \cdots, X_{n}^{+}=X_{n}$ if $X_{n} \geqq 0$, $X_{n}^{+}=0$ if $X_{n}<0$. We then have $X_{n}^{-}=X_{n}^{+}-X_{n} ; \mu^{+}=\mathscr{E} X_{n}^{+}$and $\mu^{-}=$ $\mathscr{E} X_{n}^{-}$, both necessarily finite because $\mathscr{E}\left|X_{n}\right|<\infty ; S_{n}^{+}=X_{1}^{+}+\cdots+X_{n}^{+}$, and so on. We then have $(R \gg \mu)$,

$$
\begin{aligned}
\int_{B_{n}(R)}\left|\frac{S_{n}}{n}\right| d P & =\int_{\substack{B_{n}(R) \\
S_{n}^{+} \geqq S_{n}^{-}}}\left|\frac{S_{n}}{n}\right| d P+\int_{\substack{B_{n}(R) \\
S_{n}^{-}>S_{n}^{+}}}\left|\frac{S_{n}}{n}\right| d P \\
& \leqq \int_{S_{n}^{+} \geqq n\left(R_{+} \mu\right)} \frac{S_{n}^{+}}{n} d P+\int_{S_{n}^{-} \geqq n(R-\mu)} \frac{S_{n}^{-}}{n} d P .
\end{aligned}
$$

By the weak law of large numbers, for any $\varepsilon>0$

so that

$$
P\left\{\left|S_{n}^{+} / n-\mu^{+}\right| \leqq \varepsilon\right\} \rightarrow 1,
$$

$$
\liminf _{n \rightarrow \infty} \int_{\left|S_{n}^{+} / n-\mu^{+}\right| \leqq \varepsilon} \frac{S_{n}^{+}}{n} d P \geqq\left(\mu^{+}-\varepsilon\right) .
$$

But $\mathscr{E}\left(S_{n}^{+} / n\right)=\mu^{+}$, and hence, for $(R+\mu)>\mu^{+}+\varepsilon$,

$$
\limsup _{n \rightarrow \infty} \int_{S_{n}^{+} \geq n(R+\mu)} \frac{S_{n}^{+}}{n} d P \leqq \varepsilon .
$$

The arbitrariness of $\varepsilon$ tells us that

$$
\int_{S_{n}^{+} \geqq n(R+\mu)} \frac{S_{n}^{+}}{n} d P \rightarrow 0, \quad n \rightarrow \infty .
$$

A similar argument will deal with the second integral in (3.9) and show that

$$
\int_{B_{n}(R)}\left|\frac{S_{n}}{n}\right| d P \rightarrow 0,
$$$$
n \rightarrow \infty \text {. }
$$ 
This result coupled with the observation that $L(\overline{R-\mu n}) \leqq L(\mu n)$, for $R \gg \mu$, enables us to deduce from (3.8) that

$$
\Delta_{\mathbf{0}}(R, n) \rightarrow 0, \quad n \rightarrow \infty,
$$

for $R$ sufficiently large. The proof that $\mathscr{E} M\left(\left|S_{n}\right|\right)=0(M(n \mu))$ now follows easily from our inequality (3.6) with zero substituted for $\boldsymbol{\nu}$.

Thus hypothesis $\mathscr{H}_{0}$ holds and consequently so does $\mathscr{H}_{v}$. We shall now use the weak law of large numbers to infer that, for any $\varepsilon>0$, $P\left\{A_{n}(\varepsilon)\right\} \rightarrow 1$ as $n \rightarrow \infty$. We then have, writing $\sim A_{n}(\varepsilon)$ for the event which is complementary to $A_{n}(\varepsilon)$.

$$
\begin{aligned}
\int_{\sim B_{n}(R) \cap \sim A_{n}(\varepsilon)} \frac{M_{\nu}\left(\left|S_{n}\right|\right)}{M_{\nu}(n \mu)} d P & \leqq \frac{M_{\nu}(\overline{R+\mu} n)}{M_{\nu}(n \mu)} P\left\{\sim A_{n}(\varepsilon)\right\} \\
& \leqq K_{4}\left(\frac{R+\mu}{\mu}\right)^{(v+1)} P\left\{\sim A_{n}(\varepsilon)\right\}
\end{aligned}
$$

by an argument we have already used. Therefore, using the fact that $\Delta_{\nu}(R, n) \rightarrow \infty$ as $n \rightarrow \infty$, we can infer that

$$
\mathscr{E}\left|S_{n}\right|^{\nu} M\left(\left|S_{n}\right|\right)=\int_{A_{n}(\varepsilon)}\left|S_{n}\right|^{\nu} M\left(\left|S_{n}\right|\right) d P+o\left(M_{\nu}(n \mu)\right) .
$$

We first deduce from (3.10) that

$$
\begin{aligned}
\lim \sup _{n \rightarrow \infty} \frac{\mathscr{E}\left|S_{n}\right|^{\nu} M\left(\left|S_{n}\right|\right)}{(n \mu)^{\nu} M(n \mu)} & \leqq \limsup \frac{(\mu+\varepsilon)^{(\nu+1)} L(\overline{\mu+\varepsilon n})}{\mu^{(\nu+1)} L(\mu n)} \\
& \leqq 1,
\end{aligned}
$$

in view of the arbitrariness of $\varepsilon$ and Lemma 2.2. We secondly deduce from (3.10) that

$$
\begin{aligned}
\liminf _{n \rightarrow \infty} & \frac{\mathscr{E}\left|S_{n}\right|^{\nu} M\left(\left|S_{n}\right|\right)}{(n \mu)^{\nu} M(n \mu)} \\
& \geqq \liminf _{n \rightarrow \infty} \frac{(\mu-\varepsilon)^{(\nu+1)} L(\overline{\mu-\varepsilon n})}{\mu^{(\nu+1)} L(\mu n)} P\left\{A_{n}(\varepsilon)\right\} \\
& \geqq 1,
\end{aligned}
$$

by the arbitrariness of $\varepsilon$ and the weak law of large numbers. The theorem follows from (3.11) and (3.12).

Finally we must deal with the case $v=0$ and $\mathscr{E} X_{n}=\infty$. We have, by the monotonicity of $M(x)$,

$$
\begin{aligned}
M\left(\left|S_{n}\right|\right) & \leqq M\left(\left|X_{1}\right|+\cdots+\left|X_{n}\right|\right) \\
& =\left\{\left|X_{1}\right|-\cdots+\left|X_{n}\right|\right\}\left\{\frac{M\left(\left|X_{1}\right|+\cdots+\left|X_{n}\right|\right)}{\left|X_{1}\right|+\cdots+\left|X_{n}\right|}\right\} .
\end{aligned}
$$


But, since $M(x) / x$ may be supposed non-increasing for all $x \geqq 0$, we have inequalities like

$$
\left|X_{1}\right|\left\{\frac{M\left(\left|X_{1}\right|+\cdots+\left|X_{n}\right|\right)}{\left|X_{1}\right|+\cdots+\left|X_{n}\right|}\right\} \leqq\left|X_{1}\right|\left\{\frac{M\left(\left|X_{1}\right|\right)}{\left|X_{1}\right|}\right\}
$$

and thus discover that

$$
M\left(\left|S_{n}\right|\right) \leqq M\left(\left|X_{1}\right|\right)+\cdots+M\left(\left|X_{n}\right|\right) .
$$

Hence, if $\mathscr{E} M\left(\left|X_{1}\right|\right)<\infty$, it follows that $\mathscr{E} M\left(\left|S_{n}\right|\right)=0(n)$ and the theorem is complete.

It will be clear from our proof that Corollary 2.1 is also established.

\section{Proof of Theorem 3}

Let $k$ be the least integer such that $c_{k}>0$. Then it follows from the assumption $A \in \mathscr{D}(v, M)$ that $B_{k} \in \mathscr{D}(v, M)$; in other words, we have

$$
\mathscr{E}\left|S_{k}\right|^{\nu} M\left(\left|S_{k}\right|\right)<\infty \text {. }
$$

From Lemma 2.1 we then have that

$$
\mathscr{E}\left|X_{1}\right|^{\nu} M\left(\left|X_{1}\right|\right)<\infty .
$$

If we may suppose $0<\mathscr{E} X_{1}<\infty$, we can now appeal to Theorem 2 and deduce that

$$
\mathscr{E}\left|S_{n}\right|^{\nu} M\left(\left|S_{n}\right|\right) \sim(n \mu)^{\nu} M(n \mu), \quad n \rightarrow \infty .
$$

From this asymptotic relation and the assumption $A \in \mathscr{D}(\nu, M)$ we are easily led to the conclusion

$$
\sum_{n=1}^{\infty}(n \mu)^{v} M(n \mu) c_{n}<\infty
$$

The necessity part of the theorem then follows from the easily proved fact that $M(n \mu) \asymp M(n)$. It should also be obvious at this stage how to prove the sufficiency part of the theorem.

Suppose now that $v=0$ and $\mu=\mathscr{E} X_{n}=\infty$; suppose further that we are given that (1.5) and (1.6) are true. Then, from (1.5),

$$
\mathscr{E} M\left(\left|X_{1}\right|\right)<\infty,
$$

and then, from the last part of Theorem 2, we infer that

$$
\mathscr{E} M\left(\left|S_{n}\right|\right)=0(n) .
$$

We may then conclude from (1.6) that

$$
\sum c_{n} \mathscr{E} M\left(\left|S_{n}\right|\right)<\infty,
$$

which is (1.4), the desired result. 


\section{Some results on random walks; proof of Theorem 1}

We continue the assumption that $\left\{X_{n}\right\}$ is a sequence of independent and identically distributed random variables; we write $F(x)=P\left\{X_{1} \leqq x\right\}$ for their common d.f. and $S_{n}=X_{1}+\cdots+X_{n}$, as usual, for the partial sums. We suppose $0<\mathscr{E} X_{1} \leqq+\infty$.

The following Theorem $\mathrm{A}$ is an easy consequence of Theorem 6 of Smith (1967). In that paper it is supposed that $\mathscr{E} X_{1}$ is finite whereas we are here making no such assumption. If $\mathscr{E} X_{1}=+\infty$, however, a suitable truncation argument suggests itself readily. It will be interesting to see how much is a consequence of this Theorem A.

Theorem A. Let $\Phi(x) \in \mathscr{M}^{*}$ and suppose

$$
\int_{-\infty}^{0}|x| \Phi(|x|) d F(x)<\infty .
$$

If, for any constant $\gamma<\mathscr{E} X_{1}$, we write

$$
A(x)=\sum_{n=1}^{\infty} \frac{1}{n} P\left\{S_{n}-n \gamma \leqq x\right\},
$$

then $A(0)<\infty$ and

$$
\int_{-\infty}^{0} \Phi(|x|) d \Lambda(x)<\infty
$$

If $\mathscr{E} X_{1}=\infty$ we may take $\gamma$ arbitrarily large.

We note that $\Lambda(x)$ is obviously a non-decreasing function of $x \leqq 0$ and that $\Phi(|x|) \Lambda(x) \rightarrow 0$ as $x \rightarrow-\infty$. Thus, after an integration by parts, the conclusion of Theorem A can be rewritten:

$$
\int_{-\infty}^{0} \Lambda(x)|d \Phi(|x|)|<\infty
$$

This means

$$
\sum_{n=1}^{\infty} \frac{1}{n} \int_{-\infty}^{0} P\left\{S_{n}-n \gamma \leqq x\right\}|d \Phi(|x|)|<\infty
$$

so that

$$
\sum_{n=1}^{\infty} \frac{1}{n} \int_{-n \gamma}^{0} P\left\{S_{n}-n \gamma \leqq x\right\}|d \Phi(|x|)|<\infty
$$

and $a$ fortiori

$$
\sum_{n=1}^{\infty} \frac{\Phi(n \gamma)-\Phi(0)}{n} P\left\{S_{n} \leqq 0\right\}<\infty
$$

Let $M_{\nu}(x)$ represent $x^{\nu} M(x)$ in $\mathscr{M}^{*}$, where $M(x)$ is as defined in the 
enunciation of Theorem 1. Then we deduce from Theorem A and (5.1) the following:

Result 1. (1.1) implies (1.2).

Elsewhere in Smith (1967) it is shown that the assumption $\mathscr{E} X_{1}>0$ implies the existence of constants $\rho>0, \eta>0$, such that

$$
P\left\{S_{n} \leqq 0\right\} \geqq \rho n P\left\{X_{1} \leqq-n \eta\right\} .
$$

Thus, if (1.2) holds, we must have

$$
\sum_{n=1}^{\infty} M_{\nu}(2 n \eta) P\left\{X_{1} \leqq-n \eta\right\}<\infty,
$$

since $M_{\nu}(2 n \eta) \asymp M_{\nu}(n)$ as $n \rightarrow \infty$. Therefore

$$
\sum_{n=1}^{\infty}\left\{\sum_{r=1}^{n} M_{\nu}(2 r \eta)\right\} P\left\{-(n+1) \eta<X_{1} \leqq-n \eta\right\}<\infty .
$$

But, for large $n$,

$$
\sum_{r=1}^{n} M_{\nu}(2 r \eta) \geqq \frac{1}{2}(n-1) \eta M_{\nu}(n \eta) .
$$

Hence

$$
\sum_{n=1}^{\infty} n \eta M_{\nu}(n \eta) P\left\{-(n+1) \eta<X_{1} \leqq-n \eta\right\}<\infty .
$$

We have therefore established:

Result 2. (1.2) implies (1.1).

Let us now define, for $n=1,2, \cdots$,

$$
\begin{aligned}
T_{n} & =\max \left\{S_{1}, S_{2}, \cdots, S_{n}\right\} \\
\pi_{n} & =P\left\{T_{n} \leqq 0\right\} \\
\lambda_{n} & =\frac{1}{n} P\left\{S_{n} \leqq 0\right\} .
\end{aligned}
$$

Then a familiar identity of Spitzer (1956) states that, for $0 \leqq s<1$,

$$
1+\sum_{n=1}^{\infty} \pi_{n} s^{n}=\exp \sum_{n=1}^{\infty} \lambda_{n} s^{n} .
$$

From this identity, on comparing coefficients, it is clear that $\pi_{n} \geqq \lambda_{n}$ for all $n$. Thus we have:

Result 3. (1.3) implies (1.2).

Finally, suppose again that (1.2) holds. Then we can set 


$$
\Lambda=\sum_{n=1}^{\infty} \lambda_{n}<\infty
$$

and

$$
\beta(s)=\sum_{n=1}^{\infty}\left(\lambda_{n} \Lambda^{-1}\right) s^{n}, \quad 0 \leqq s \leqq 1,
$$

will be a probability generating function in the class $\mathscr{G}(\nu, M)$. Furthermore

$$
\gamma(s)=e^{A(s-1)}
$$

is the familiar Poisson probability generating function and $\gamma(s)$ is plainly a member of $\mathscr{G}(\nu, M)$. From Corollary 3.1 we can infer that $\gamma(\beta(s))$ is another member of $\mathscr{G}(v, M)$ if only we can be sure that the 'mean' of $\beta(s)$ is finite, i.e. that

$$
\sum_{n=1}^{\infty} n \lambda_{n}<\infty
$$

This is the same as the statement

$$
\sum_{n=1}^{\infty} P\left\{S_{n} \leqq 0\right\}<\infty .
$$

When $v \geqq 1,(5.3)$ is a consequence of (1.2), which we are assuming true. Thus, when $v \geqq 1$, we have.

Result 4. (1.2) implies (1.3).

Finally, we must also prove Result 4 when $v=0$. In this case (5.3) is, in general, false. However, if we expand the Poisson generating function $\gamma(s)=\sum_{0}^{\infty} c_{n} s^{n}$ it is obviously true that $\sum_{1}^{\infty} n c_{n}<\infty$. Thus the case $\nu=0$ of Corollary 3.1 completes the proof of Result 4 .

Theorem 1 follows from Results 1, 2, 3, and 4.

\section{References}

[1] C. C. Heyde (1964). Two probability theorems and their application to some first passage problems. J. Austral. Math. Soc., 4, 214-222.

[2] W. L. Smith (1967). A theorem on functions of characteristic functions and its application to some renewal theoretic random walk problems, Proceedings of the Fifth Berkely Symposium on Mathematical Statistics and Probability, Vol. II. Part 2, pp. 265-309, Berkeley, University of California Press.

[3] W. L. Smith and W. E. Wilkinson (1965), Proceedings of the Symposium on Congestion Theory, Chapel Hill, University of North Carolina Press.

[4] F. Spitzer (1956), A combinatorial lemma and its application to probability theory, Trans. Amer. Math. Soc., 82, 323-339.

University of North Carolina

Chapel Hill, N.C. 